\title{
A Scaled Effective Medium Theory for Calculation of Resonant and Relaxation Frequency in Magnetic Composites
}

\author{
Ricky Lamar Moore \\ Georgia Tech Research Institute, Georgia Institute of Technology, Atlanta, United States
}

Email address:

rick.moore@gtri.gatech.edu

\section{To cite this article:}

Ricky Lamar Moore. A Scaled Effective Medium Theory for Calculation of Resonant and Relaxation Frequency in Magnetic Composites. American Journal of Physics and Applications. Vol. 9, No. 1, 2021, pp. 15-24. doi: 10.11648/j.ajpa.20210901.13

Received: December 6, 2020; Accepted: December 14, 2020; Published: January 22, 2021

\begin{abstract}
Scaled Effective Medium Theory (ScEMT) is applied to predict magnetic resonant and relaxation frequency in polymer-magnetic particle composites with favorable comparison to measured data. A single scaling function is identified that uses magnetic particulate resonant and relaxation frequencies, or magnetization and anisotropy field, volume fraction and DC susceptibility as predicted by ScEMT. Previous publications demonstrated that ScEMT improved the prediction of DC susceptibility as compared to classical models. Maxwell-Garnett (MGT) and Coherent Model Approximation (CMA) serve as theoretical baselines for comparison. However, both require separate scaling functions in their prediction of resonant and relaxation. Measured data are presented that suggest a single scaling function of ScEMT is sufficient to calculate both parameters. The paper emphasizes the application of the models and shows a wide range of particulate chemistries. ScEMT calculates susceptibility, resonant and relaxation frequency that agree with measurement. The paper concludes by predicting dispersive permeability that represents improvement over both CMA and MGT models. Future studies will address formulation of an EMT model (s) that describe mixtures of hard and soft magnetic materials mixed in a polymer composite. EMT for composite dielectric properties will be expanded to address the chaining. Early results of that effort will be reported in a separate paper.
\end{abstract}

Keywords: Effective Medium Theory, Permeability, Frequency Dispersions

\section{Introduction}

Composites formed as mixtures of magnetic and nonmagnetic materials are applied in a wide range of modern RF technologies. New ferrite compositions, such as those for bio-magnetics, are in constant development and these provide new candidates for forming new ferrofluids; materials to reduce electromagnetic interference; application in wideband antennas and microwave devices such as isolators. Example applications are listed [1, 2]. Composites with desired electromagnetic frequency dispersive parameters have historically been developed by experimentation using an iterative process of formulation-measurement-formulationmeasurement, etc. This procedure introduces a latency in the design process for identifying optimized material formulations and purely empirical approaches can investigate only a limited parameter space. This lag time and additional effort can be reduced and parameter spaces fully investigated by using accurate predictive models of electromagnetic mixture parameters.

This paper is an extension of the previously developed and experimentally tested volumetrically scaled effective medium theory (ScEMT) [3, 4]. ScEMT demonstrated significant improvements in the prediction of low frequency composite magnetic susceptibility as compared to other effective medium models and preliminary results showed promise for predicting composite resonant frequency. In the current work, the predictive accuracies of ScEMT, MGT and CMA, models are further tested [4-6]. The models are applied to predict functionality and magnitude of resonant and relaxation frequency in composites containing magnetic particulate volume fractions from 10 to near $100 \%$. It is shown that the 
ScEMT's ability to predict DC susceptibility can be translated to improved prediction in frequency parameters and thus an improved prediction in frequency dispersive susceptibility. Knowledge of DC susceptibility, resonant frequency and relaxation parameter (time) can be applied in Landau-Lifschitz-Gilbert models. which can then be exercised to make composite formulation trades and optimize frequency dispersive composite designs for application without having to fabricate and empirically test each candidate formulation (LLG) [7].

This paper begins with a short review of ScEMT and comparisons to MGT [5], CMA [6] and LLG [7]. The three models' predictions of resonant and relaxation frequency are compared to measured data for a range of ferrites. MGT and CMA serve as theoretical baselines of comparison. MGT and CMA use different power laws in particulate volume fraction and susceptibility, to predict resonance and relaxation. Measured data are presented that argue for a single power low. The ScEMT scaling of resonant and relaxation frequency uses a single scaling parameter that predicts both frequency parameters of composite with good accuracy.

\section{ScEMT Review}

ScEMT is built upon the Bruggeman effective medium theory, BEMT [8]. BEMT was modified to include a nonlinear dependence of magnetic demagnetization factor on volume fraction. The approach is supported by publications by Chevalier and Le Floc'h M. Anhalt, et al., C. Alvarez and S. H. L. Klapp J. L. Mattei and collaborators, [9-14].

Magnetic constitutive equations for the CMA, BEMT and MGT describe two-phase composites, Equations 1-3. CMA and MGT equations have been simplified from their standard format by specializing them for nonmagnetic matrices. Subscripts $e, p$ and $m$ indicate composite, particulate and matrix parameters respectively with $P_{p}$ being volume fraction of the particulate, and $\chi_{\mathrm{p}}$ as the particulate's magnetic susceptibility. $A_{B}$ is demagnetization factor which is $1 / 3$ for spherical particulates.

$$
\begin{gathered}
C M A: \chi_{e}=\frac{P_{p}^{1 / 3} \chi_{p}}{1+\left(1-P_{p}^{1 / 3}\right) \chi_{p}}=\frac{P_{p} \chi_{p}}{P_{p}^{1 / 3}\left(1+\chi_{p}\right)-P_{p}^{1 / 3} \chi_{p}} \\
M G T: \chi_{e}=\frac{3 P_{p} \chi_{p}}{\left(3+\chi_{p}\right)-P_{p} \chi_{p}} \\
B E M T: P_{p} \frac{\mu_{e}-\mu_{p}}{\mu_{e}+\left(\mu_{p}-\mu_{e}\right) A_{B}}+\left(1-P_{p}\right) \frac{\mu_{e}-\mu_{m}}{\mu_{e}+\left(\mu_{m}-\mu_{e}\right) A_{B}}=0
\end{gathered}
$$

The BEMT equation for spherical particulates describes a binary system whose microstructure changes from differentiated to symmetric near a volume fraction of $1 / 3$, the percolation threshold $P_{C}$. J. P. Clerc, et al. notes that when $A_{B}=1 / 3, \mu_{e}$ in the denominator of Equation 3 becomes multiplied by the factor 2 [15]. In general, the multiplier is $d-1$ where $d$ is system dimensionality $(d-1=2$ in $3 \mathrm{D}$ and $d-1=1$ in 2D). It represents demagnetization due to composite morphology. Dimensionality is connected to the percolation threshold by $P_{C} \cong d^{-1}$.

Concepts in percolation theory suggest insight into a nonlinear coupling between demagnetization, geometry, particulate volume fraction, particle-matrix magnetic properties and percolation threshold [15]. In a composite, the largest cluster size $S\left(a, P_{p}, P_{c}\right)$ is approximately

$$
S\left(a, P_{p}, P_{c}\right)=a\left(P_{p}-P_{c}\right)^{-\gamma}
$$

where $a$ is the characteristic dimension of a single particle and $\gamma$ is a non-integer exponent that changes with dimension of the composite. Demagnetization is a function of magnetic cluster size and shape. That physics combined with Equation 4 implies that demagnetization has a non-linear dependence on volume fraction.

Demagnetization in a composite also has a magnetic component. Particulate and matrix are assumed to have different susceptibilities. Magnetic poles are formed at particulate-matrix interfaces and produce an opposing internal demagnetization field within particulates. Formation of poles and demagnetization changes with particulate agglomerate shape, size and magnetic coupling that produces chaining of magnetic particulates. Chaining was demonstrated to have large impacts on permeability in measurements of [16]. In summary, demagnetization is a function of particulate susceptibility, particulate cluster sizes and shapes, and the media susceptibility that surrounds the particulate. This complex dependence supplies second need for nonlinearity in the system as demagnetization is itself a function of composite susceptibility, which changes with particulate volume fraction, etc.

Numerical simulation, and cluster experiments were used to identify a geometrical based function, $A_{p}$, that related an average particulate cluster size to demagnetization via volume fraction of [3]. $A_{p}$ replaces the classical BEMT demagnetization constant, $d-1=\frac{1-P_{c}}{P_{c}}$, by the ratio $A_{p}=$ $\frac{1-A_{C}}{A_{C}}$, to produce the ScEMT equation,

$$
\text { ScEMT: } P_{p} \frac{\mu_{e}-\mu_{p}}{A_{p} \mu_{e}+\mu_{p}}+\left(1-P_{p}\right) \frac{\mu_{e}-\mu_{m}}{A_{p} \mu_{e}+\mu_{m}}=0
$$

where

$$
A_{c}=A_{0}\left\{1 .-A_{1}\left(1-P_{p}\right)^{\gamma}\right\} \text { and } A_{p}=\left(\frac{1-A_{C}}{A_{C}}\right)
$$

These equations express one relationship between volume fraction, cluster size and percolation threshold via its relationship to demagnetization. Magnetic particulate percolation (i.e. clustering) is not random but is biased by internal magnetic fields that couple particulate poles. Strong coupling between particulates increases probability of particulate chaining which reduces the overall system energy especially for particle susceptibilities of large magnitude.

The free parameters, $A_{0}, A_{1}$ and $\gamma$ were determined by fitting the ScEMT Equation 5 to measurement of nondispersive, low frequency $(<10 \mathrm{MHz})$ susceptibilities of composites made from $10-40 \mu m$, multi-domain $\mathrm{Ni}_{0.31} \mathrm{Zn}$ 
${ }_{0.58} \mathrm{Cu}_{0.08} \mathrm{~F}_{2.03} \mathrm{O}_{4}$. Samples were made with ferrite volume concentrations of $18,20,21,30,32,45,57$ and $64 \%,[3,4]$. A $100 \%$ dense control sample was also measured and found DC susceptibility of 863 . The $100 \%$ data supplied the base input for model testing. Composite and solid samples were cut to size for a $7 \mathrm{~mm}$ coaxial transmission line test fixture. Complex reflection and transmission measurements were measured and from those permittivity and permeability were calculated. Those data were then fit to a LandauLifshitz-Gilbert resonance equation. DC susceptibility, resonant frequency and relaxation parameters could be inferred from that fit. Details of measurement and procedures can be found in along with measured data of the $100 \%$ dense ferrite [17].

The fitted values for the ScEMT scaling function are near unity with numerical values of: $\mathrm{A}_{0}=0.975, \mathrm{~A}_{1}=0.923$ and $\gamma=1.210$, when rounded to the nearest thousandth. Even though parameter values from one data fit the model has been successfully applied to a wide range of ferrite chemistries.

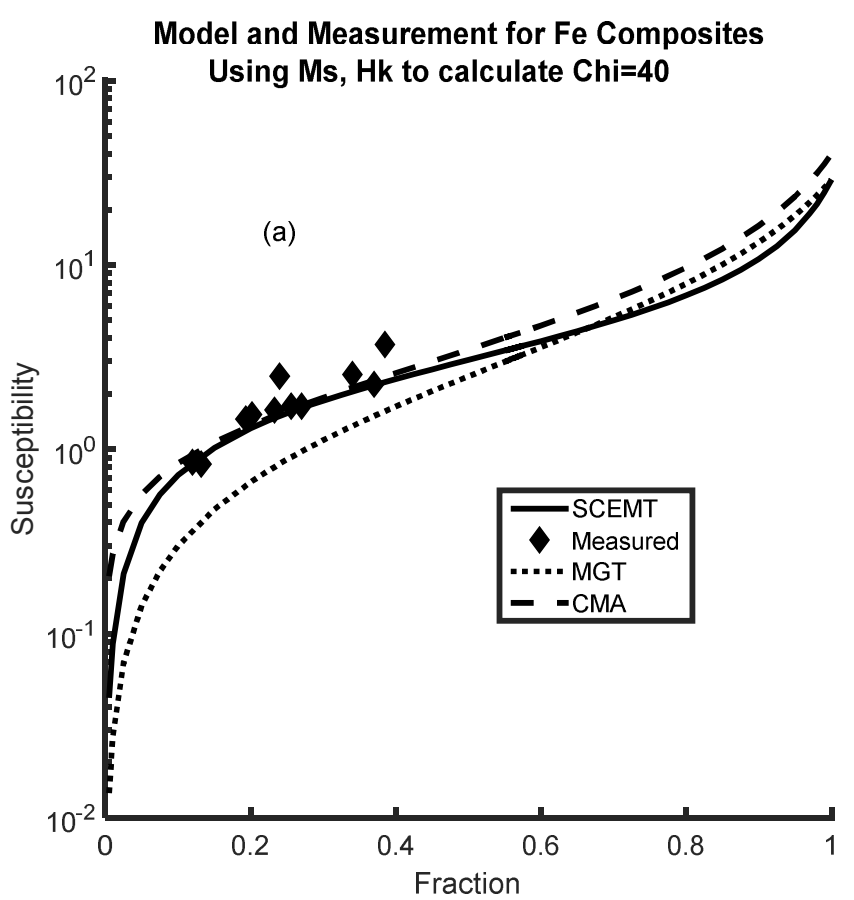

Magnetic data for 12 ferrite and ferromagnetic particulates were found in publications and used in this study. These were: 1. NiZn, MnZn Ferrite industrial powders [22, 23], 2. Mn $\mathrm{Zn}_{.41} \mathrm{Fe}_{2.06} \mathrm{O}_{4}$, [26], 3. $\mathrm{Ni}_{.24} \mathrm{Zn}_{.65} \mathrm{Fe}_{2.04} \mathrm{O}_{4}$ [26], 4. $\mathrm{Fe}_{3} \mathrm{O}_{4}[24-$ 27], 5. $\mathrm{Ni} \mathrm{Mn}{ }_{.02} \mathrm{Fe}_{1.9} \mathrm{O}_{4}$ [26], 6. (MnO) ${ }_{.25}(\mathrm{ZnO})_{.2115} \mathrm{Fe}_{2} \mathrm{O}_{3}$ [27] 7. $\mathrm{BaCoZn} \mathrm{Fe}_{16} \mathrm{O}_{37}$ [28], 8. NiFe, e.g. $\mathrm{Fe}_{53} \mathrm{Ni}_{47}$, [29, 34, 18], 9. Fe, [30-33], 10. Ni ${ }_{.36} \mathrm{Zn}_{.64} \mathrm{Fe}_{2} \mathrm{O}_{4}$, [34]. 11. $\mathrm{Ni}_{.7} \mathrm{Zn}_{3}$ $\mathrm{Fe}_{2} \mathrm{O}_{4},[7,9], 12 . \mathrm{Ni}_{.3} \mathrm{Zn}_{.58} \mathrm{Cu}_{.08} \mathrm{Fe}_{2.03} \mathrm{O}_{4}$ and $\mathrm{Ni}_{.24} \mathrm{Zn}_{.65}$ $\mathrm{Cu}_{.07} \mathrm{Fe}_{2.04} \mathrm{O}_{4},[17,35]$.

The majority of publications included information on bulk susceptibility, resonant, relaxation frequencies and composite microstructure. Figure 1 show examples of ScEMT predicted and measured susceptibility. ScEMT susceptibility predictions continue to show better agreement with measurement than BEMT, MGT and CMA models at mid volume fractions (10 $100 \%$ ) and large susceptibility particulates.

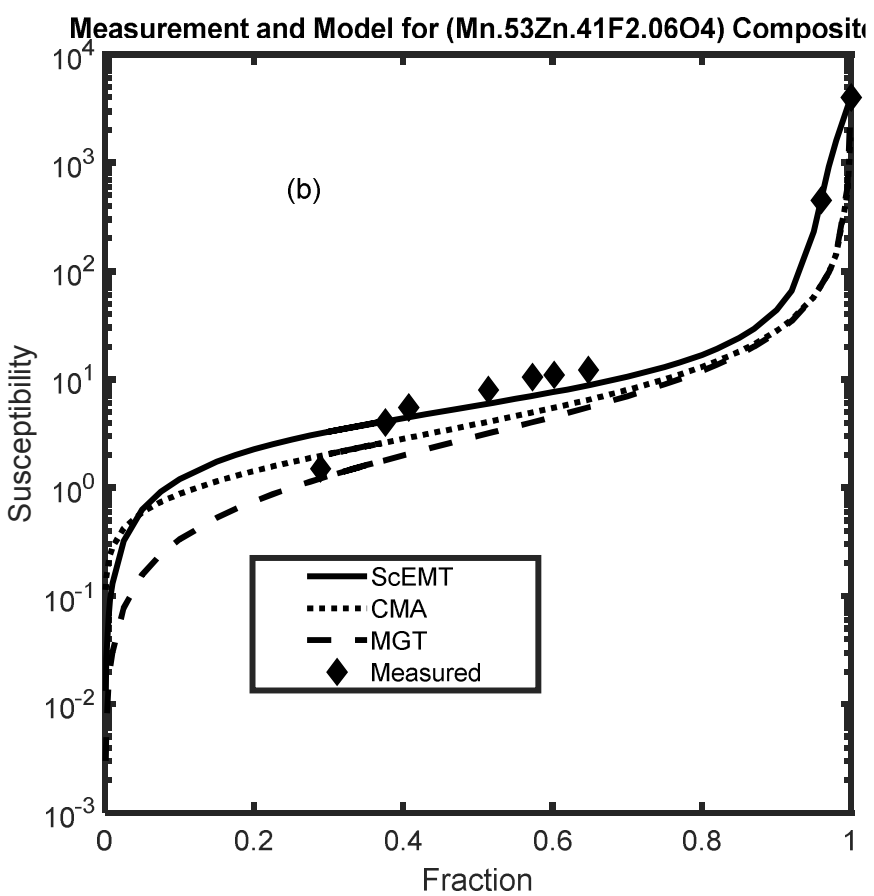

Figure 1. EMT model predictions for (a) left Fe particulate and (b) right $\mathrm{Mn}_{.53} \mathrm{Zn}_{.41} \mathrm{Fe}_{2.06} \mathrm{O}_{4}$ composites. Fe data were taken from a range of commercial EMC products. The $\mathrm{Mn}_{.53} \mathrm{Zn}_{.41} \mathrm{Fe}_{2.06} \mathrm{O}_{4}$ has a DC permeability near 4000.

\section{Composite Resonant Frequency and Relaxation Time Constants}

In the case of negligible external field, the frequency dispersive ferrite or ferrite - polymer composite LLG model is given in Equation 7. It uses the DC susceptibility of particulate, subscript $p$, or composite subscript $c$. There is also an anisotropy field and a relaxation factor.

$$
\begin{aligned}
\chi_{p(\omega)} & =\frac{\chi_{p, 0}\left\{1 .+j \alpha_{p} \omega /\left(\gamma H_{k, p}\right)\right\}}{1-\left(\frac{\omega}{\gamma H_{k, p}}\right)^{2}+j 2 \alpha_{p} \omega /\left(\gamma H_{k, p}\right)} . \\
\chi_{c(\omega)} & =\frac{\chi_{c, 0}\left\{1 .+j \alpha_{c, p} \omega /\left(\gamma H_{k, c}\right)\right\}}{1-\left(\frac{\omega}{\gamma H_{k, c}}\right)^{2}+j 2 \alpha_{c, p} \omega /\left(\gamma H_{k, c}\right)}
\end{aligned}
$$

$H_{k, c, p}$ is the anisotropy field of composite or particle; $\chi_{c, p, 0} \quad$ is the DC susceptibility of composite and particle, $\omega$ is radial 
frequency at which properties are calculated. The parameter combination $\frac{\alpha_{c, p}}{\left(\gamma H_{k, c}\right)}$, is a relaxation parameter or relaxation time. Its inverse is a relaxation frequency and is proportional to the $1 / 2$ width of the imaginary part of the susceptibility at resonance.

It is assumed that bulk susceptibility, resonant and relaxation frequency will be available as input to any calculations. Accurate prediction of composite susceptibility is the first step in calculating frequency dispersive spectra of mixtures. That first step uses the ScEMT model, Equation 5. The second and third steps are prediction of resonant frequency and relaxation frequency. Their calculation leverages the improved accuracy of ScEMT.

The prediction of composite resonance, and relaxation frequency, are approached by insertion of an analytical equation for composite susceptibility (i.e. MGT or CMA) in the LLG equation [6]. The resulting dispersive models take forms that easily identify equations for composite resonant frequency, $f_{r c}$ and relaxation, $f_{d c}$ where both $f_{r c}$ and $f_{d c}$ are proportional to particulate bulk resonance $f_{r 0}$ and relaxation $f_{d 0}$. The MGT and CMA derived relations for the resonance and relaxation parameters are shown in Equations 8-11. Both equations and ScEMT, support experimental observations that magnetic particulates placed in a nonmagnetic matrix, produce materials whose resonance is shifted to a higher frequency compared to that of the particulate. This reflects physics of internal field formation arising from the many poles set up at particle-particle and particle-matrix interfaces. However, when compared to experiment Equations 8 and 10 often under predict the volume fraction induced shift in composite resonant frequency and DC susceptibility magnitude for particulates with large magnitude susceptibility.

$$
\begin{aligned}
& C M A[6]: \frac{f_{r c}}{f_{r 0}}=\left\{1 .+\chi_{p}\left(1-P_{p^{3}}^{\frac{1}{3}}\right)\right\}^{1 / 2} \\
& C M A[6]: \frac{f_{d c}}{f_{d 0}}=\left\{1 .+\chi_{p}\left(1-P_{p^{3}}^{\frac{1}{3}}\right)\right\}
\end{aligned}
$$

And

$$
\begin{gathered}
M G T[6]: \frac{f_{r c}}{f_{r 0}}=\left\{1 .+\chi_{p}\left(1-P_{p}\right)\right\}^{1 / 2} \\
M G T[6]: \frac{f_{d c}}{f_{d 0}}=\left\{1 .+\frac{\chi_{p}\left(1-P_{p}\right)}{3}\right\}
\end{gathered}
$$

Though the arguments $1 .+\chi_{p}\left(1-P_{p}\right)$ and $1 .+\chi_{p}\left(1-P_{p^{\frac{1}{3}}}\right)$ are both used in calculating resonance and relaxation frequencies, the power laws used in calculation of resonance and relaxation differ. Resonant frequency contains a square root and relaxation is linear. CMA and MGT also differ in their dependence on volume fraction; CMA using the $1 / 3$ power and MGT is linear in volume fraction. The model volume fraction scales are not consistent with measured data for many magnetic polymer composites.

Figure 2 shows resonant and relaxation frequency as functions of magnetic particulate volume fraction for two ferrite composites. For much of the volume range, below $(60 \%)$ the logarithmic plot of relaxation and resonance frequency are parallel when plotted versus volume fraction. The data suggests that nearly identical functional forms can be used to predict both resonant and relaxation. The differing $1 / 2$ power and linear dependence proposed in Equations 8-11 are counter to this observation.

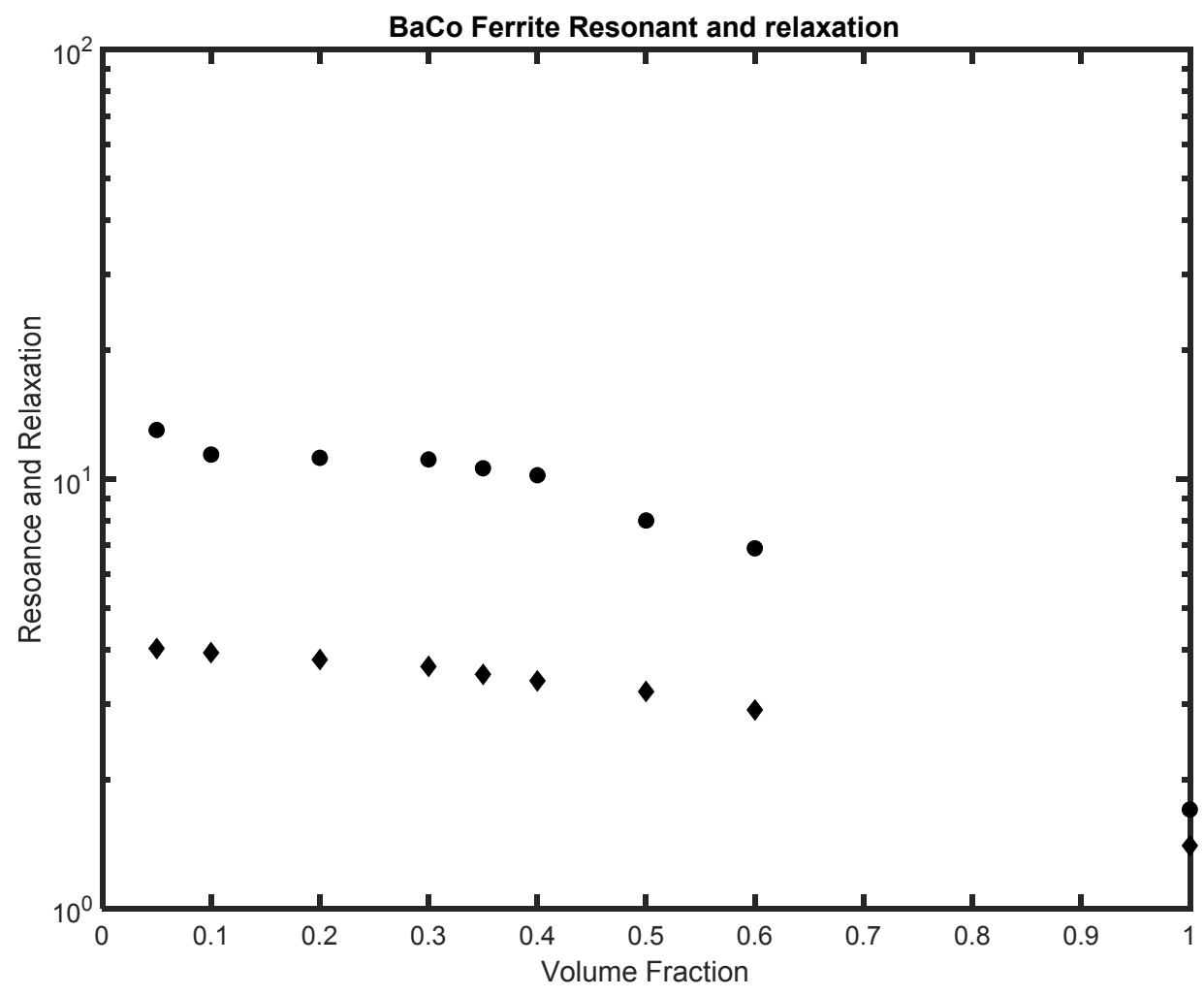




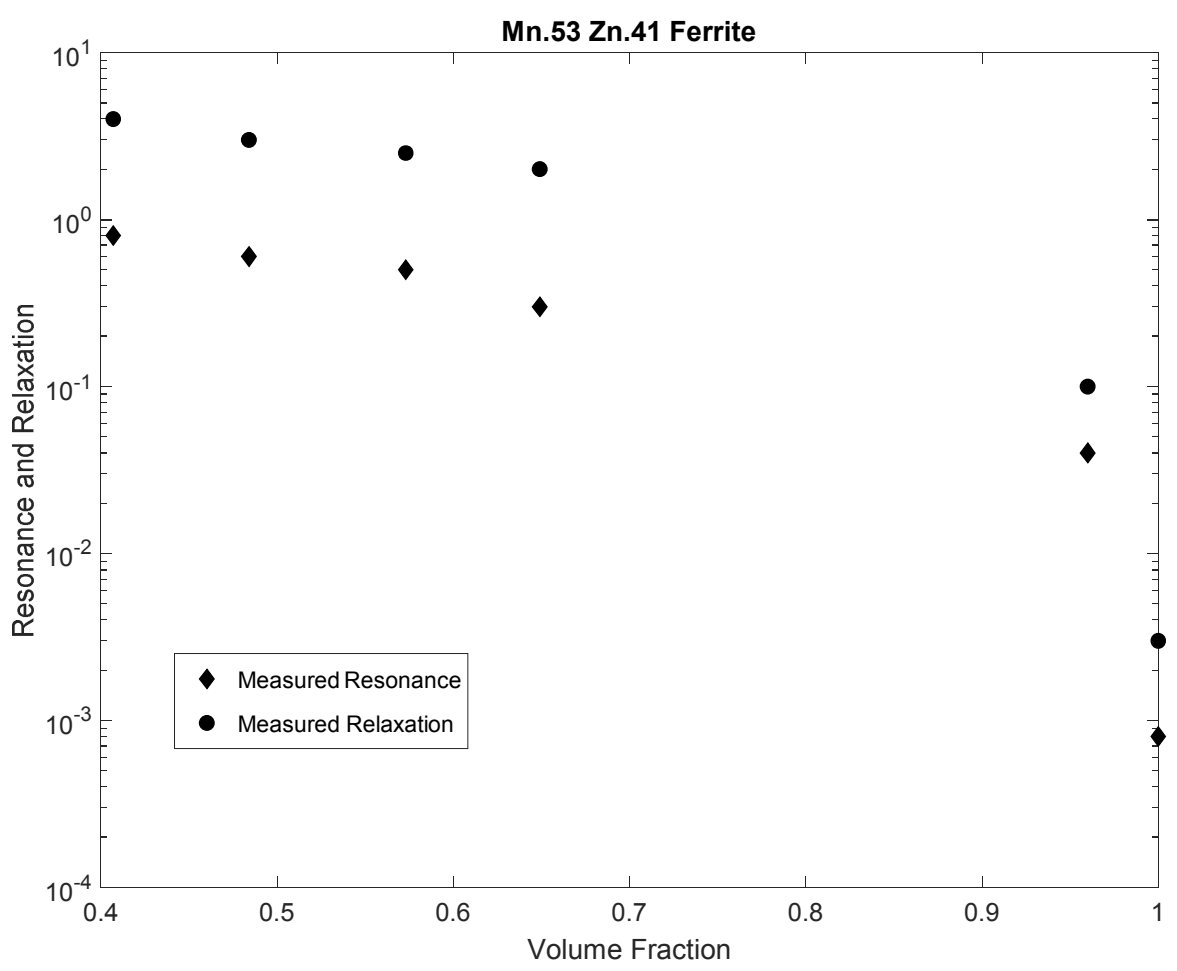

Figure 2. Plots of measured resonance frequency and relaxation for a BaCo ferrite (upper figure) and lower figure Mn.53 Zn. ${ }_{41}$ Ferrite.

Rozanov, Koledintseva et al. took another approach to determine resonance, $[19,20]$. The authors apply integral relationships that leverage Snoek's law [21]. The ScEMT resonance and relaxation model shown below also applies Snoek's law but applies DC susceptibility as calculated from ScEMT.

A form of Snoek's law should apply for multidomain particulate isotropic composites such as those that are topics of this of this paper. Thus it is assumed that the product of susceptibility and resonant frequency are equal to a constant multiplied by the material magnetization, i.e. $\chi_{p} \omega_{r}=4 \pi M s$ where $\mathrm{Ms}$ is the magnetization and $\chi_{p}$ is the bulk susceptibility or $\chi_{p} \omega_{r}=C$ where $C$ is a constant.

$\chi_{p} \omega_{r}$

$=4 \pi M s, M s$ is magnetization and $\chi_{p}$ is bulk suseptibility or $\chi_{p} \omega_{r}$ $=C$ where $C$ is a constant.

We assume that composite bulk value of magnetization can be linearly scaled with volume fraction. Simple algebra shows composite susceptibility, $\chi_{\mathrm{c}}$, will be related to composite relaxation frequency, $\omega_{r, c}$ bulk susceptibility, $\chi_{p}$, volume fraction, $\mathrm{P}_{\mathrm{p}}$, and bulk resonance frequency, $\omega_{r}$, by,

$$
\omega_{r c}=\frac{P_{p} \omega_{r}}{\chi_{c}} \chi_{p}
$$

The biggest impact of ScEMT in the calculation is the improved prediction of $\chi_{c}$. Since ScEMT predicts susceptibility better than other EMT in the mid- to highvolume fraction range, it is not surprising that improvements are apparent for other magnetic parameters like resonance frequency.

A small algebraic modification facilitates the step toward calculation of relaxation frequency. The composite should exhibit an anisotropy field, $H_{k, c}$, that is also assumed proportional to composite bulk resonant frequency, $\omega_{r, c}$. The effective anisotropy field of the composite can be calculated from

$$
\gamma H_{k, c}=\omega_{r c}=\frac{P_{p} \omega_{r p}}{\chi_{c}} \chi_{p} .
$$

In Equation 12 the DC susceptibility, volume fraction and resonance of the particle, $\chi_{p, 0}, P_{p}$ and $\omega_{r p}$ are known from bulk data. Composite susceptibility, $\chi_{c .0}$ is predicted from ScEMT. In practice ScEMT has improved predictions other EMT, for large susceptibility particulates in the $10-100 \%$ volume fraction range. In Equation 7 we note that the composite anisotropy field is a denominator in both resonant and relaxation contributions. Equation 12 supplies that parameter for composites.

Empirical evidence, i.e. Figure 1, suggested that resonance and relaxation appear to scale with volume fraction. That guides an initial test for an equation for relaxation. We use the ansatz that composite relaxation parameter, $\alpha$ will be proportional to that of the particle in the bulk. It is assumed constant and can be determined from the ratio of resonant to relaxation frequency in the bulk.

$$
\alpha_{c}=\frac{\beta \alpha_{p}}{\gamma H_{k, c}}=\alpha_{p} \frac{\chi_{c}}{P_{p} \omega_{r, p} \chi_{p}}
$$

With these substitutions the equation for dispersive susceptibility becomes

$$
\chi_{c(\omega)}=\frac{\chi_{c}\left\{1 .+j \omega \alpha_{p} \frac{\chi_{c}}{P_{p} \omega_{r, p \chi_{p}}}\right\}}{1-\left(\frac{\omega \chi_{c}}{P_{p} \omega_{r, p} \chi_{p}}\right)^{2}+j 2 \omega \alpha_{p} \frac{\chi_{c}}{P_{p} \omega_{r, p} \chi_{p}}}
$$


The model is tested by using measured dispersive susceptibility data. Data were taken from publications on the twelve magnetic composites presented in the previous section. Each composite had a single magnetic compound distributed uniformly in a nonmagnetic polymer and thus made an isotropic mixture. Ferrite particulates had susceptibilities that arranged from $10-5000$. Figures 3 and 4 are representative of prediction and measurement. Figure 5 shows an Equation 14 prediction of permeability for four volume fractions of the
Figure $4 \mathrm{NiZnCu}$ ferrite.

Since MGT and CMA predictions are often close, only CMA is shown for comparison to ScEMT. ScEMT is most accurate in the prediction of resonant frequency (Equations 8 and 10) and especially for composites using a pigment with large susceptibility values. The NiZnCuFerrite plot is the only one in which digital data were available. Other data were acquired by reading values from plots.
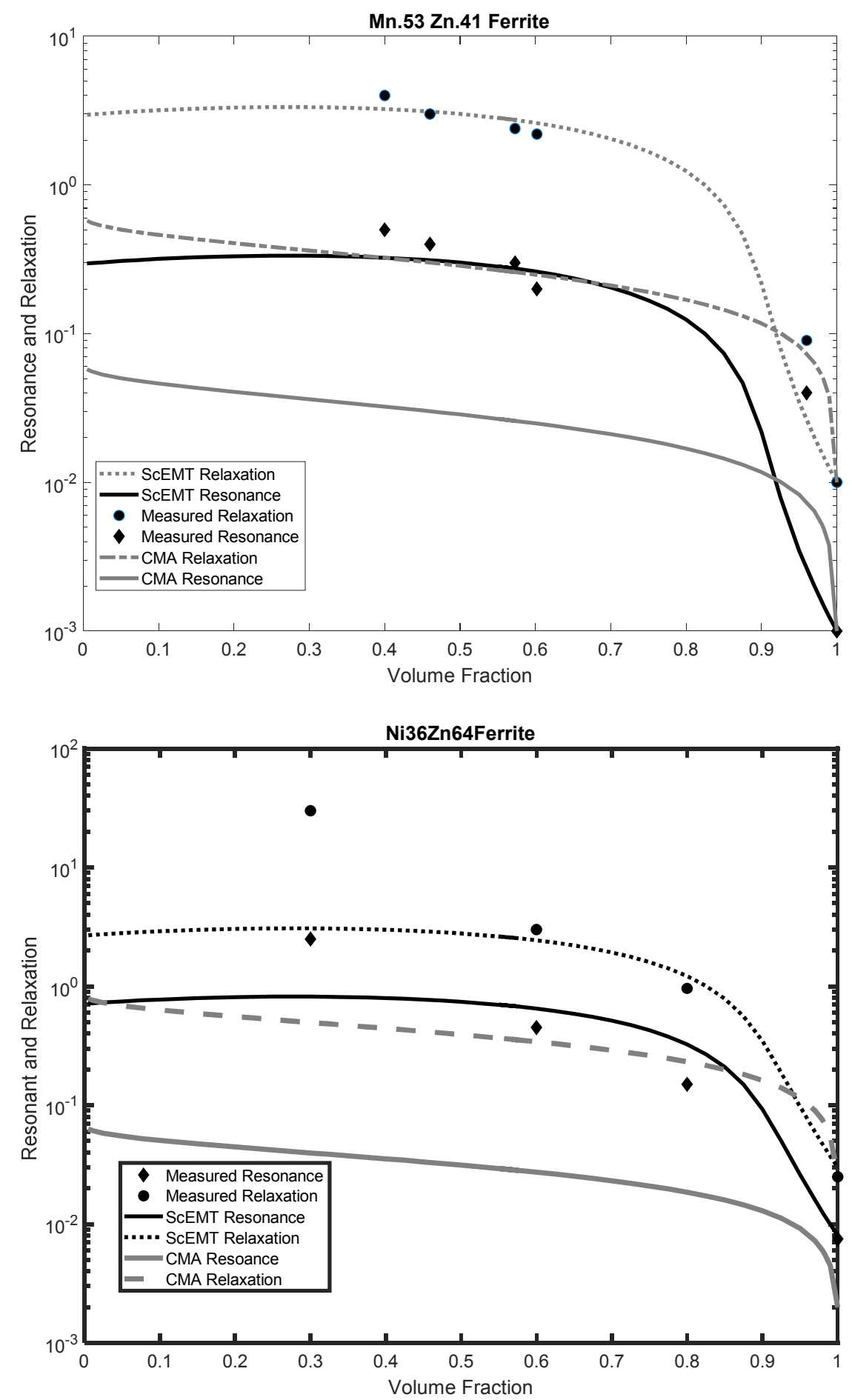

Figure 3. ScEMT, and CMA model predictions for measured resonant and relaxation frequency of composites. (a) upper figure is composites of susceptibility of 4000 and (b) lower figure is with susceptibility 1200. 

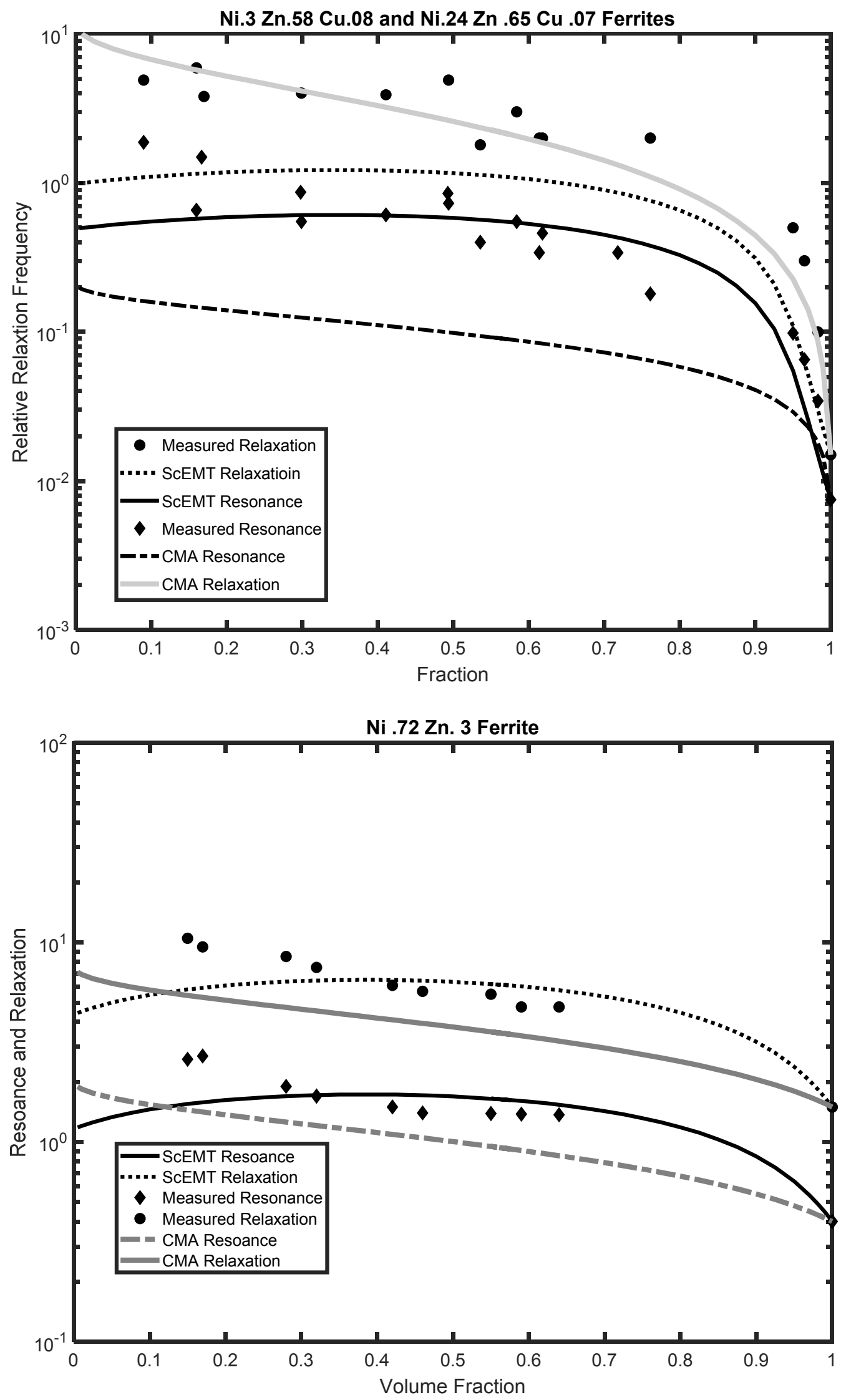

Figure 4. ScEMT and CMA model predictions compared to measured resonant and relaxation frequency of composites for high susceptibility particulates: (a) upper figure susceptibility 863 and (b) lower figure susceptibility is 20. In this case, CMA predicts the relaxation better than ScEMT; however, its resonant prediction is poor. 


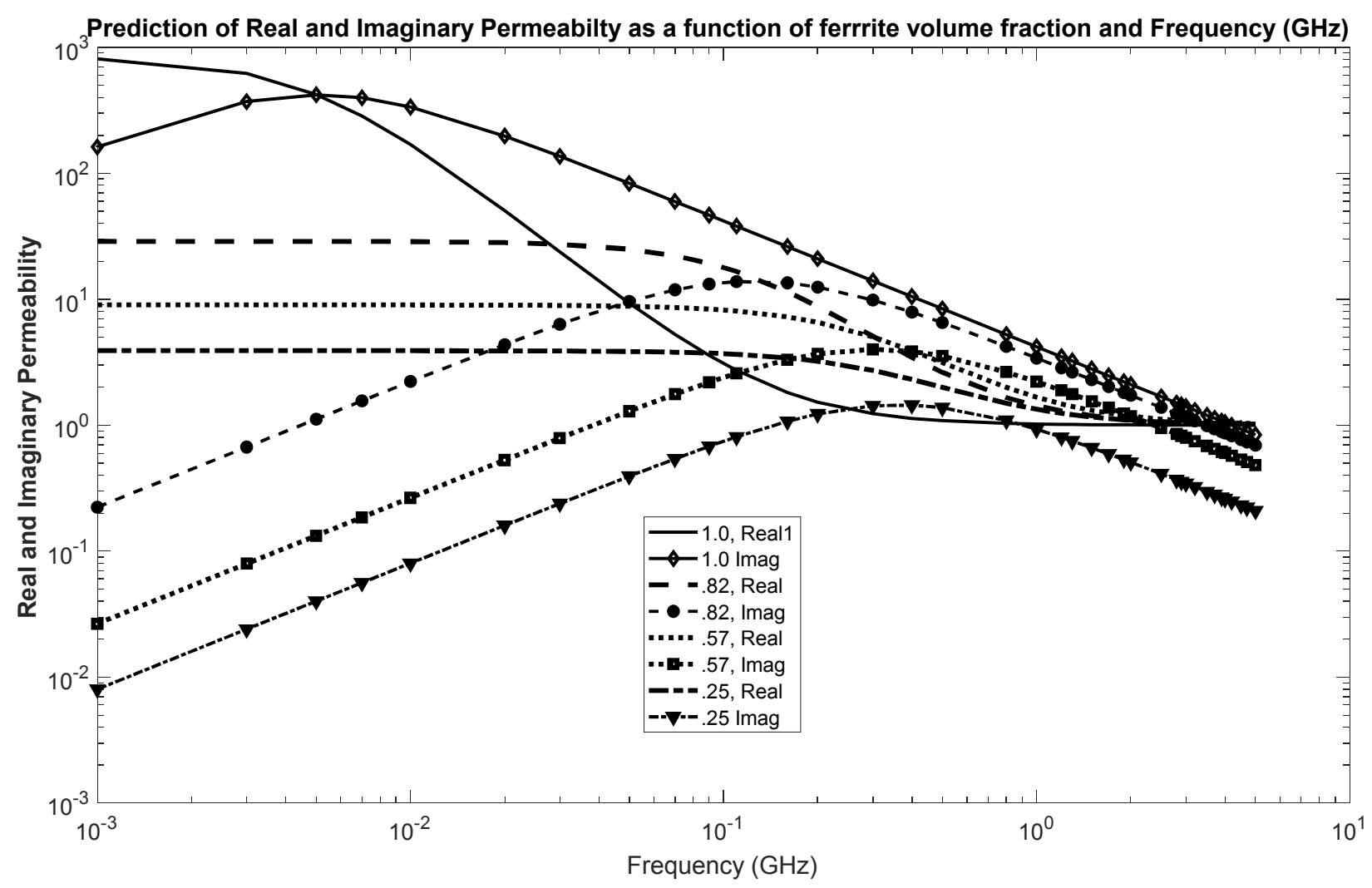

Figure 5. The figure shows an example prediction of composite permeability where NiZnCu ferrite pigment of Figure 3 has been used at volume fractions of $1.0,82,57$ and.25.

Figure 6 brings the ScEMT modeled parameters together to illustrate the frequency and volumetric prediction of complex permeability. The model produces the characteristic increase in resonant and broadening of the resonance as magnetic particle fraction is reduced. Figure 6 is a comparison of Equation 14 predictions using two data sets. The ScEMT prediction applies parameters calculated from Equations 5, 12 and 14. The real "Data Fit" uses the measured values of DC permeability, resonant and relaxation frequencies for the same chemistry ferrite composite. Volume fraction is $30 \%$. Agreement is typical of ferrite composites studied in this effort.

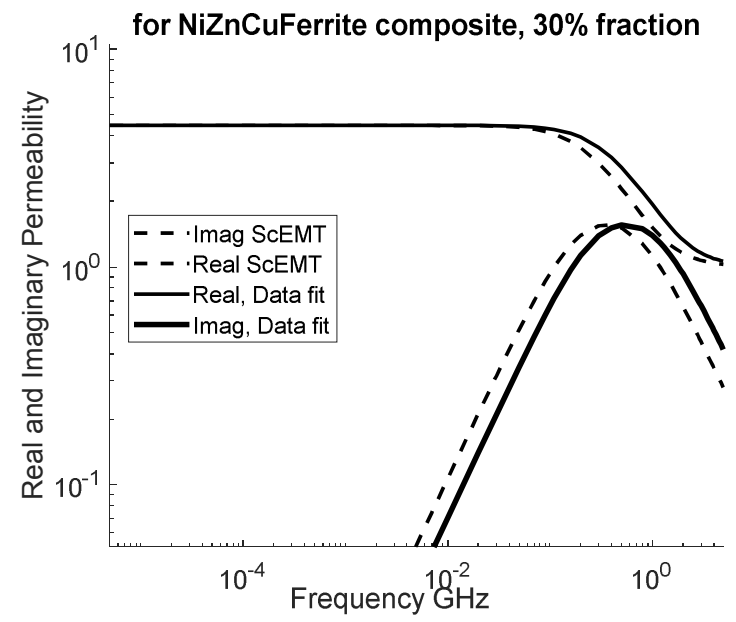

Figure 6. ScEMT and predicted and measured dispersive permeability.

\section{Conclusions and Path Forward}

Maxwell-Garnett Theory (MGT), Coherent Model Approximation (CMA), and Scaled Effective Medium Theory (ScEMT) were applied in this paper to predict magnetic susceptibility, resonant and relaxation frequency in polymer-magnetic particle composites. Model and measurement comparisons of resonant frequency were followed by model and measurement comparisons for relaxation frequency. CMA, MGT and ScEMT with various volumetric scales are used in the relaxation frequency study. Though ScEMT susceptibility, resonant and relaxation are not perfect in prediction of measurement, they demonstrate significant improvement over other historical EMT in composites that use particulates with large susceptibility. The improved accuracy of ScEMT for large susceptibilities is supported by numerical studies [11]. The publications suggest that spherical particulates with large magnetization and large susceptibility, are more likely to attract one another and their attraction will exceed frictional drag of media surrounding the particles. Particles assemble into chains and large structures and therefore volume fraction scaled models are necessary to reflect the fundamental physics. This physics is reflected in the ScEMT demagnetization scaling. Recent and future studies will be shifted to formulating an EMT model which describes mixtures of hard and soft magnetic materials mixed in a polymer composite. Early results of that effort will be reported in a separate paper. 


\section{References}

[1] Périgo E. A., et al. (2018) Past, present, and future of soft magnetic composites, Appl. Phys. Rev. 5, 031301 (2018); https://doi.org/10.1063/1.5027045.

[2] Idris Fadzidah Mohd, et al., (2015) Recent developments of smart electromagnetic absorbers based polymer-composites at gigahertz frequencies, J. Mag. Mag. Mat., 405, 197, doi.org/10.1016/j.jmmm.2015.12.070.

[3] Moore R. L. (2019) Development of a volume fraction scaling function for demagnetization factors in effective media theories of magnetic composites, AIP Advances 9, 035107; https://doi.org/10.1063/1.5078736.

[4] Moore R. L. (2019) Development and test of concentration scaled demagnetization in effective media theories of magnetic composites, J. Appl. Phys. 125, 085101 (2019); https://doi.org/10.1063/1.5053791.

[5] Maxwell-Garnett J. C., (1904) Colours in Metal Glasses and in Metallic Films, (1904) Philosophical Transactions the Royal Society A, Vol. 203, No. 359-371, 1904, pp. 385-420. doi: 10.1098/rsta.1904.0024 T.

[6] Tsutaoka t., et al. (2013) Analysis of the Permeability Spectra of Spinel Ferrite Composites Using Mixing Rules, 2013 IEEE EMC International Symposium Digest,. 545, IEEE-978-14799-0409-9/13.

[7] Lax B., Button K. J., (1962), Microwave Ferrites and Ferrimagnetics, 474-477, McGraw Hill, Library Congress Catalog 62-11947 36785.

[8] Bruggeman D. A. G. (1935), Berechnung verschiedener physikalischer Konstanten von heterogenen Substanzen. Dielektrizitätskonstanten und Leitfähigkeiten der Mischkörper ausisotropen Substanzen, Ann. Phys. 24, 636 doi.org/10.1002/andp.19354160705.

[9] Chevalier A., Le Floc'h M. Dynamic permeability in soft magnetic composite materials Journal of Applied Physics 90, 3462 (2001); doi.org/10.1063/1.1389520.

[10] Anhalt M., Weidenfeller B. (2007) Magnetic properties of polymer bonded soft magnetic particles for various filler fractions Journal of Applied Physics 101, 023907 (2007); https://doi.org/10.1063/1.2424395.

[11] Alvarez C., Klapp S. H. L., (2012) Percolation and orientational ordering in systems of magnetic nanorods, Soft Matter, 2012, 8, 7480, DOI: 10.1039/c2sm25636c.

[12] J. L. Mattei J. L., et al., (2000) Gyroresonance in unsaturated composite bodies: Experiments and theory, Journal of $\begin{array}{llll}\text { Applied } & \text { Physics } & \text { 87, } & 4975\end{array}$ https://doi.org/10.1063/1.373220.

[13] Chevcalier A., Le Floc'h M., (2003) Dynamic permeability in soft magnetic composite materials, J. of Applied Physics, 90, 3462, (2001); https://doi.org/10.1063/1.1389520.

[14] Alexis Chevalier A. Mattei, J. L., M. Le Floc'H, M, (2000). Ferromagnetic resonance of isotropic heterogeneous magnetic materials: theory and experiments. J. of Mag. and Mag. Mat. Elsevier, 2000, Vol. 215, pp. 66. https://hal.univ0brest.fr/hal00491965.
[15] J. P. Clerc J. P., Giraud G. Laugier J. M., J. M. Luck, (1990) The electrical conductivity of binary disordered systems, percolation clusters, fractals and related models Advances in Physics, 39, 3, 191-309, https://doi.org/10.1080/00018739000101501.

[16] Y. Shimada, et al, (2011) Amorphous Submicron Particle Chains With High Permeability, IEEE TRANSACTIONS ON MAGNETICS, VOL. 47, NO. 10, OCTOBER 2011, DOI: 10.1109/TMAG.2011.2151181.

[17] Moore R. L., Electromagnetic Composites Handbook: Models, Measurement and Characterization, Chapter 12, McGraw Hill ISBN: 978-1-25-958504-3 2016T.

[18] T. Kasagi, et al., (1998) High Frequency Permeability of Perm alloy and its Composite Materials, Journal of The Magnetics Society of Japan, b1.22, Supplement, No. S1 (1998), https://doi.org/10.3379/jmsjmag.22.S1_295.

[19] Rozanov K. N., et al., (2005) Microwave permeability of Co2Z composites, Journal of Applied Physics 97, 013905 (2005); https://doi.org/10.1063/1.1827911.

[20] Rozanov K. N., Koledintseva M. Y., (2016) Application of generalized Snoek's law over a finite frequency range: A case study, J. Appl. Phys. 119, 073901 (2016); https://doi.org/10.1063/1.4941844.

[21] J. L. Snoek, (1948) DISPERSION AND ABSORPTION IN MAGNETIC FERRITES AT FREQUENCIES ABOVE ONE Mc/s Physica, Volume 14, Issue 4, May 1948, Pages 207-217, https://doi.org/10.1016/0031-8914(48)90038-X.

[22] Fiske J. J., et al., (1997) Percolation in magnetic composites. Journal of Materials Science 32, 5551-5560 (1997). https://doi.org/10.1023/A:1018620407013.

[23] Tsutaoka T. (2003) Frequency dispersion of complex permeability in $\mathrm{Mn}-\mathrm{Zn}$ and $\mathrm{Ni}-\mathrm{Zn}$ spinel ferrites and their composite materials Journal of Applied Physics 93, 2789 (2003); https://doi.org/10.1063/1.1542651.

[24] Zheng Hong, et al., (2009) Microwave Magnetic Permeability of $\mathrm{Fe}_{3} \mathrm{O}_{4}$ Nanoparticles Chin. Phys. Lett. » 2009, Vol. 26 Issue (1): 017501 DOI: 10.1088/0256-307X/26/1/017501.

[25] Liong S., PhD Thesis, "A Multifunctional Approach to Development, Fabrication, and Characterization of $\mathrm{Fe} 3 \mathrm{O} 4$ Composites" School of Material Science and Engineering, Georgia Institute of Technology, (2005).

[26] Pippin J. E. and C. L. Hogan C. L., "Initial Permeability Spectra of Ferrites and Garnets", Sci. Rept. No. 1, AF19 (604) 1084, AFCRC-TN-59-169, Harvard University 1959.

[27] Babayan V., et al., (2012) Magneto active feature of in-situ polymerized polyaniline film developed on the surface of manganese-zinc ferrite, Applied Surface Science 258 (2012) http://dx.doi.org/10.1016/j.apsusc.2012.04.12.

[28] Li Z. W. Et al., (2008) Characteristics of effective permeability and resonance frequency for barium ferrite/epoxy composites, J. Appl. Phys. 103, 073901 (2008); https://doi.org/10.1063/1.2899958.

[29] Massango H. et al., (2016) Electromagnetic properties of $\mathrm{Fe} 53 \mathrm{Ni} 47$ and $\mathrm{Fe} 53 \mathrm{Ni47/Cu}$ granular composite materials in the microwave range, Mater. Res. Express 3 (2016) 095801 doi: 10.1088/2053-1591/3/9/095801. 
[30] Wu L. Z., et al., (2006), High frequency complex permeability of iron particles in a nonmagnetic matrix, J. Appl. Phys. 99, 083905 (2006); https://doi.org/10.1063/1.2190719.

[31] Shimada Y. et al., (2007) Permeability of submicron and nanometer ferromagnetic particle composites J. Appl. Phys. 101, 09M505 (2007); https://doi.org/10.1063/1.2712019.

[32] Ramprasad R., et al., (2004) Magnetic properties of metallic ferromagnetic nanoparticle composites, Journal of Applied Physics 96, 519 (2004); https://doi.org/10.1063/1.1759073.

[33] Slama J., Gruskova A., (2007) Modification of Frequency
Spectra of Ferrite Composites Sept. 10-12, 2007, Pilsen Czech Republic, https://core.ac.uk/download/pdf/295587653.pdf.

[34] Chih-Wen Chen, Magnetism and Metallurgy of Soft Magnetic Materials, Dover Publications (December 8, 2011) ISBN 13: 9780486649979.

[35] Nakamura T. Tsutaoka T., Hatakeyama, K. (1994) Frequency dispersion of permeability in ferrite composite materials, Journal of Magnetism and Magnetic Materials Volume 138, Issue 3, December 1994, Pages 319-328 https://doi.org/10.1016/0304-8853(94)90054-X. 